Documentation et bibliothèques

DOCUMENTATION BIBLIOTHËQUES

\title{
LE CODOC REMANIÉ : LE TRAITEMENT DES PUBLICATIONS OFFICIELLES À L'ÉCOLE POLYTECHNIQUE DE MONTRÉAL
}

\section{Olivier Paradis}

Volume 26, numéro 4, décembre 1980

URI : https://id.erudit.org/iderudit/1054217ar

DOI : https://doi.org/10.7202/1054217ar

Aller au sommaire du numéro

Éditeur(s)

Association pour l'avancement des sciences et des techniques de la documentation (ASTED)

\section{ISSN}

0315-2340 (imprimé)

2291-8949 (numérique)

Découvrir la revue

Citer cet article

Paradis, O. (1980). LE CODOC REMANIÉ : LE TRAITEMENT DES PUBLICATIONS OFFICIELLES À L'ÉCOLE POLYTECHNIQUE DE MONTRÉAL. Documentation et bibliothèques, 26(4), 211-214. https://doi.org/10.7202/1054217ar

Tous droits réservés (C) Association pour l'avancement des sciences et des techniques de la documentation (ASTED), 1980
Ce document est protégé par la loi sur le droit d'auteur. L'utilisation des services d'Érudit (y compris la reproduction) est assujettie à sa politique d'utilisation que vous pouvez consulter en ligne.

https://apropos.erudit.org/fr/usagers/politique-dutilisation/ 


\section{chronique \\ delarecherche}

\section{LE CODOC REMANIÉ: LE TRAITEMENT DES PUBLICATIONS OFFICIELLES À L'ÉCOLE POLYTECHNIQUE DE MONTRÉAL}

La bibliothèque de l'École polytechnique devenait en 1977 la deuxième bibliothèque universitaire québécoise à adhérer au groupe CODOC ontarien après celle de l'Université de Sherbrooke, UTLAS devant servir d'intermédiaire pour la transmission des données.

Le système CODOC est un système automatisé conçu pour le traitement local, en différé, des publications gouvernementales ou du même type. Traitement qui se veut simple, rapide, économique, régi par un minimum de règles catalographiques et reposant sur une structure de notice catalographique unique, quel que soit le document à traiter, de sorte que l'entrée principale, par exemple, est toujours faite à l'organisme qui publie ou diffuse le document, les auteurs personnels apparaissant comme entrées secondaires seulement.

Le système CODOC comprend comme deuxième élément majeur un système ordinal de numérotation qui reflète l'organisation même des organismes concernés. Cette numérotation a été conçue à partir du principe que les publications gouvernementales peuvent être organisées et exploitées plus facilement et avec plus de profit lorsqu'elles sont constituées en collection séparée et qu'elles sont classées logiquement selon l'ordre même des ministères, divisions, etc. dont elles sont issues. Cette numérotation de 23 caractères permet de regrouper les documents, d'abord par pays, et ensuite par province ou état, ministère, subdivision, collection, numéro de document et langue.

Travailler avec le système CODOC, c'est aussi, si l'on est également membre du groupe CODOC, participer à la production d'un fichier collectif. Réalisé grâce à la fusion mensuelle des fichiers locaux des membres, ce fichier, disponible sur microfiches, permet de tirer lors des fusions mensuelles subséquentes une copie de toutes les notices choisies pour le traitement en dérivé et de les faire verser dans le fichier local.

Enfin, le système CODOC offre, en plus des accès habituels par auteurs, titres et collections, un accès par sujets à l'aide d'un index permuté KWOC. Nous expliquerons plus loin pourquoi nous n'avons jamais utilisé cet index à la bibliothèque de l'École polytechnique.

\section{Avantages et inconvénients}

Un des premiers avantages de CODOC, c'est sans contredit sa grande simplicité. Le traitement des documents peut habituellement être fait de façon 
expéditive tout en conservant à la notice catalographique tous les points d'accès habituels propres à une notice plus élaborée.

Le fait d'utiliser un numéro de classement pour la cote plutôt qu'un indice de classification rend le processus d'attribution de la cote à chaque document extrêmement rapide et facile une fois que le numéro de base pour l'organisme ou la collection a été attribué.

Le fait, par ailleurs, de faire partie du groupe CODOC nous a permis de dériver du fichier collectif plus du tiers du nombre total de notices créées, ce qui a allégé d'autant notre travail.

Quant à toutes les autres notices que nous avons dû créer et coder, nous avons pu en faciliter considérablement le codage et la saisie au terminal en utilisant la commande "Mémoire" de CATSS pour la saisie de tous les éléments communs à plusieurs notices comme c'est le cas lorsque nous traitons en bloc tous les documents d'une même collection.

Si les avantages de cette approche étaient appréciables, les inconvénients se sont révélés finalement encore plus importants. Disons d'abord que la simplicité même du traitément, qui avait pu sembler avantageuse au départ, s'est avérée très vite un embarras sérieux surtout pour certains types de documents, tels les périodiques. De même le fait de coder les notices en format MARC mais sans pouvoir en utiliser toutes les possibilités à cause des contraintes de traduction du format MARC à $C O D O C$ rendait ce travail frustrant.

L'emploi obligatoire des majuscules, alors qu'il n'aurait été guère plus difficile d'employer également les minuscules, en est un exemple.

Notre participation à CODOC entraînait aussi pour nous des coûts annuels de plus en plus élevés sans que nous puissions par ailleurs entrevoir d'amélioration dans les délais inacceptables rencontrés dans le processus de dérivation des notices du fichier collectif. Ces délais signifiaient que I'on pouvait rarement envisager de traiter en dérivé un document récent, ce qui diminuait d'autant les avantages de l'aspect collectif du système.

Ces inconvénients de CODOC ne nous auraient peut-être pas amenés à l'abandon de ce système n'eût été souvent la très grande disparité de la forme des entrées pour un même organisme. En effet, en vertu des normes de traitement propres à CODOC, un même organisme pouvait être entré, selon le cas, en anglais ou en français, au long ou avec le sigle seulement, et cela, toujours en majuscules, de sorte qu'il n'était pas rare de trouver dans notre fichier jusqu'à cinq entrées différentes pour un même organisme (ex.: INTERNATIONAL ATOMIC ENERGY AGENCY, AGENCE INTERNATIONALE DE L'ÉNERGIE ATOMIQUE, IAEA, AIEA pour les documents traités dans CODOC, et Agence internationale de l'énergie atomique pour les documents traités en dehors de CODOC, en format MARC complet). Une telle disparité nous apparaissait inacceptable. Comme la correction à cette situation ne semblait pas pouvoir venir de l'intérieur de CODOC qui se montrait, contrairement à nos espérances, fermé à toute modification dans le sens d'une plus grande normalisation dans ce domaine, nous n'avions pas d'autre choix que de chercher une autre voie.

II nous fallait cependant trouver une solution de rechange qui nous permettrait autant que possible de conserver les principaux avantages de l'approche CODOC tout en nous affranchissant de ses principaux inconvénients. Le système d'identification de CATSS devait, nous l'espérions, nous permettre d'y arriver.

\section{Nouvelle approche (dite à "notices brèves")}

Nous quittions en septembre 1979 le groupe CODOC avec l'intention d'utiliser le système d'identification de CATSS dès que possible, ce qui devait se faire le mois suivant. Entre-temps, déjà libérés des contraintes du système CODOC, nous avons voulu repenser en profondeur toutes les normes auxquelles nous nous étions astreints jusque-là. II s'agissait d'améliorer le catalogage et le codage des publications gouvernementales tout en conservant une 
approche normalisée et la numérotation propre à CODOC.

Un nouveau bordereau fut dessiné. Les zones fixes CODOC furent remplacées par les zones appropriées du format MARC. D'autres zones furent ajoutées. L'emploi des seules majuscules fut abandonné. Pour toutes les zones et sous-zones choisies, le format MARC et la ponctuation ISBD furent adoptés intégralement. La numérotation CODOC fut, quant à elle. conservée à peu près intacte.

Quant au problème majeur entraîné par la disparité des entrées, le système d'identification de CATSS devait nous permettre d'y remédier. Pour chaque nom d'organisme ou titre de collection à employer, nous créons un dossier d'identification dans lequel sont inclus tous les renvois de même que les anciennes formes CODOC (les anciennes formes devant servir à corriger les entrées des dossiers CODOC lors d'un balayage automatique ultérieur de notre fichier). Nous y notons aussi le numéro CODOC de base correspondant de façon à disposer au moment de traiter un document précis d'une collection de tous les renseignements nécessaires pour traiter rapidement le document. De plus, pour faciliter le codage, la vérification et la saisie des données au terminal, le numéro du dossier d'identification est substitué au nom complet dans la zone appropriée du bordereau de catalogage. On peut se rendre compte que cette nouvelle approche, tout en assurant une amélioration notable de la qualité du catalogage et du codage, n'en permet pas moins de traiter les documents d'une même collection à peu près aussi rapidement qu'auparavant, et de façon beaucoup plus méthodique et plus sûre.

Cette nouvelle approche ne s'applique cependant pas aux périodiques maintenant traités de façon élaborée par l'emploi de toutes les zones pertinentes du format MARC. Seul le numéro CODOC les distingue des périodiques traités ailleurs et classifiés selon LC.

Précisons enfin que cette nouvelle approche a aussi l'avantage de permettre l'introduction dans les fichiers SOM de tous les renvois désirés, ce qui va entraîner une amélioration substantielle de la qualité de notre "microfichier" et faciliter, nous l'espérons, son utilisation.

\section{Index permuté}

Nous n'avons pas encore parlé de l'accès "sujet". Ce problème des plus épineux pour nous, puisque depuis l'automne 1976 nous n'employons plus de vedettes-matière, même pour les publications de notre collection générale, vient de trouver sa solution. Depuis mars 1980, nous offrons aux usagers, en guise d'outil de repérage "sujet", un index permuté de type KWIC qui couvre l'ensemble des documents traités avec CATSS. Cette solution était celle qui avait été envisagée dès les débuts de l'automatisation de notre fichier bibliographique.

Avait été prévu cependant au départ un index KWOC du genre offert par CODOC. Mais un examen un peu plus attentif de ce genre d'index nous fit vite constater son inefficacité, du moins pour l'usage que nous comptions en faire. L'index de type KWIC, malgré son aspect plutôt rébarbatif, s'avéra l'outil indiqué. C'est ainsi qu'en septembre 1978 des spécifications détaillées pour un index permuté de type KWIC furent envoyées à UTLAS. C'est, à peu de choses près, le produit maintenant offert aux usagers.

\section{Premier bilan}

Nous avons maintenant codé plus de 5,000 notices d'identification, et nous commençons à ressentir les fruits de ce travail particulièrement pour le traitement des publications gouvernementales. Une première constatation résulte de cette nouvelle approche: pour les monographies d'une part, et pour les publications sériées d'autre part, il s'avère généralement plus simple, plus économique et plus rapide de faire du traitement original que de chercher à faire du traitement en dérivé. La perte de l'avantage que nous donnait CODOC de puiser dans le fichier collectif ne semble donc pas trop lourde. La rapidité d'écriture, 
de vérification et de saisie que permet la

nouvelle approche la compense amplement.

L'expérience des derniers mois

semble devoir confirmer la justesse de cette nouvelle approche. II sera intéressant, un peu plus tard, d'évaluer les déboursés additionnels et les économies entraînés par ces nouvelles procédures et ces nouvelles normes. Espérons que l'avenir confirmera qu'à coûts égaux il est maintenant possible de faire aussi sinon même plus vite qu'auparavant, et beaucoup mieux.

Olivier Paradis

Bibliothèque

École polytechnique

Université de Montréal

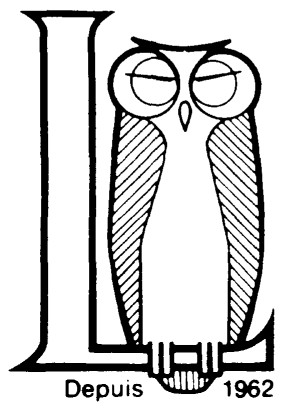

CATALOGUE 1980

BIBLIOTHẼQUES

R.M. LEDUC PROTECTEURS DE LIVRES EN FILM POLYESTER - PELlICULES AUTO-ADHÉSIVES - RUBAN DE LECTO-CONTACT OU PELLIMAT - DÉVIDOIRS - TRICOL - PROTÉGE-FICHES VERTICAL, HORIZONTAL OU SURELEVÉ - FICHES-GUIDE • PROTEGE-PÉRIODIQUES - POCHETTES DE VOLUME • POCHETTES DE VINYL - ÉTIQUETTES COLLANTES LECTO-DURO - ÉTIQUETTES AUTO-ADHÉSIVES - COLLE PLAXANOL FICHES DE CONTRÔLE - RELIEURS ACCOPRESS - ACCOGRIP - PERFORATEURS MATÉRIEL D'EXPÉDITION - RUBANS AUTOADHÉSIFS - DOSSIERS SUSPENDUS CHEMISES - ENVELOPPES PAPIER KRAFT REMBOURRE •

C.P. 1379, Marieville, Qué. JOL 1JO (514) 658-0661 